\title{
Two new species in the Micarea prasina group from Western Europe
}

\author{
Pieter P. G. VAN DEN BOOM, A. Maarten BRAND, Brian J. COPPINS \\ and Emmanuël SÉRUSIAUX
}

\begin{abstract}
Detailed morphological and anatomical studies informed by molecular inferences with $\mathrm{mtSSU}$ as a marker revealed two new species of Micarea in Western Europe, both belonging to the core group of the genus, namely the $M$. prasina group: $M$. herbarum from the Netherlands and Poland and M. meridionalis from Portugal and Italy. Micarea herbarum looks like a small or depauperate $M$. denigrata but clearly differs by the lack of gyrophoric acid, while $M$. meridionalis is distinguished by its granular thallus and the production of micareic acid.
\end{abstract}

Key words: lichen diversity, lichenized Ascomycota, molecular phylogeny, taxonomy

Accepted for publication 8 August 2016

\section{Introduction}

Recently, the study of the genus Micarea has again attracted the attention of lichenologists. This is because the production of molecular sequences and phylogenetic reconstructions has revealed greater diversity in Micarea, including in Europe and Macaronesia (Czarnota \& Guzow-Krzemińska 2010; van den Boom \& Ertz 2014; Guzow-Krzemińska et al. 2016), and because a significant number of new species continue to be described from all parts of the world (van den Boom 2010; Sérusiaux et al. 2010; Svensson \& Thor 2011; Câceres et al. 2013; Aptroot \& Câceres 2014; Brand et al. 2014; Córdova-Chávez et al. 2014; McCarthy \& Elix 2016). In Europe, the genus was investigated in a major revision (Coppins 1983) and was further studied at a smaller scale by Coppins (2009) for Great Britain and Ireland, and Czarnota (2007) for Poland.

The $M$. prasina group is known to be very variable and was suspected of containing

P. P. G. van den Boom: Arafura 16, NL-5691 JA Son, The Netherlands.

A. M. Brand: Klipperwerf 5, NL-2317 DX Leiden, The Netherlands.

B. J. Coppins: Royal Botanic Garden Edinburgh, 20A Inverleith Row, Edinburgh, EH3 5LR, UK.

E. Sérusiaux (corresponding author): Evolution and Conservation Biology Unit, University of Liège, Sart Tilman B22, B-4000 Liège, Belgium. Email: E.Serusiaux@ulg.ac.be additional species (Coppins 1983). Several studies dealing with collections within Europe (Czarnota \& Guzow-Krzemińska 2010; Guzow-Krzemińska et al. 2016) and outside Europe (Barton \& Lendemer 2014; Brand et al. 2014) have shown that it represents a complex assemblage of species that is yet to be properly disentangled. Based on morphological, anatomical, chemical and molecular evidence, our study of the material belonging to that group available to us from Europe yielded evidence of two undescribed species. They are formally described in this paper.

\section{Material and Methods}

The material examined was collected by the authors in Belgium, France, Germany, the Netherlands, Portugal, Romania and Spain (Canary Islands, Tenerife). Anatomical measurements were made on material mounted in dilute $\mathrm{KOH}$ for conidia and paraphysis width, and in water for all other characters. In each collection, $c .10$ well-developed ascospores representing the size and shape variation detected, conidia and paraphyses were measured with a precision of $0.1 \mu \mathrm{m}$ using camera lucida drawings. For thallus description, we refer to Coppins (1983) for the use of areolate- and goniocyst-type, the latter being "a finely granular thallus, composed of discrete, \pm globular structures, mostly c. $12-40 \mu \mathrm{m}$ diam., these ecorticate granules consist of clustered algal cells intertwined and surrounded by short-celled hyphae, and never protected by an amorphous covering layer".

Chemical compounds were studied using several methods: response to UV light, melting point determination, microcrystallization and thin-layer chromatography 
(TLC) using solvent system A (toluene/1,4-dioxane/acetic acid 180:45:5) for all collections and C (toluene/acetic acid 170:30) when gyrophoric acid was suspected (Huneck \& Yoshimura 1996; Orange et al. 2010), in both cases visualization of spots was achieved with sulphuric acid sprayed over the plates, followed by heating at $110^{\circ} \mathrm{C}$ for $c .5 \mathrm{~min}$.

Well-preserved specimens lacking any visible symptoms of fungal infection were selected for DNA isolation. Extraction of DNA and PCR amplification were performed following the protocol of Cubero et al. (1999). We used the primers mrSSU1 and mrSSU3R (Zoller et al. 1999) for the production of $\mathrm{mtSSU}$ sequences. Amplicons were sequenced by Macrogen ${ }^{\circledR}$. Sequence fragments were assembled with Sequencher version 5.2.3 (Gene Codes Corporation, Ann Arbor, Michigan). Sequences were then subjected to MEGABLAST searches to detect potential contamination. They were included in a single matrix together with data extracted from GenBank, published in Andersen \& Ekman (2005), Czarnota \& GuzowKrzemińska (2010), Guzow-Krzemińska et al. (2016) and van den Boom \& Ertz (2014). We chose Byssoloma leucoblepharum and B. subdiscordans as outgroup following the topology obtained for the Pilocarpaceae by Miadlikowska et al. (2014). Accession data are included in Table 1. Sequences were aligned manually using MacClade version 4.08 (Maddison \& Maddison 2005) and alignment was checked with the MAFTT software (Katoh \& Standley 2013). Ambiguous regions were delimited using the online version of Gblocks v0.91b (Castresana 2000) at http://molevol.cmima.csic.es/castresana/Gblocks. $\mathrm{html}$, allowing for gap positions within the final blocks, and carefully checked manually. The aligned matrix is available from the corresponding author on request. We inferred the maximum likelihood tree and bootstrap support values based on 1000 pseudoreplicates in the same run using RAxML HPC2 version 7.2.8 (Stamatakis 2006; Stamatakis et al. 2008) with the GTRCAT model and the default settings as implemented on the CIPRES portal (Miller et al. 2010). Phylogenetic trees were visualized using FigTree v1.2.3 (Rambaut 2009). Branch support values were considered significant when ML bootstrap (BS) $>70 \%$. We included in our accessions for molecular inferences other specimens besides those belonging to the two species described here as new, for the following reasons: 1) we wished to assess the only phylogenetic tree available for the genus with further data (Andersen \& Ekman 2005); 2) we suspect that Micarea is more complex in other groups than the prasina group and we wished to test this hypothesis.

\section{Results}

The data matrix includes $21 \mathrm{mtSSU}$ sequences newly produced for the following species: $M$. adnata, $M$. byssacea, $M$. denigrata, $M$. doliiformis, $M$. herbarum, $M$. lignaria var. lignaria, $M$. meridionalis, $M$. nowakii, M. micrococca, M. prasina, M. pycnidiophora, $M$. stipitata and $M$. viridileprosa. The single most-likely tree (Fig. 1) resolves two strongly supported clades within Micarea. Both species newly described in this paper are resolved within a single clade and more precisely within a strongly supported $(\mathrm{BS}=98)$ subclade including all accessions of the M. prasina group (Coppins 1983, 2009; Czarnota 2007; Czarnota \& GuzowKrzemińska 2010; Guzow-Krzemińska et al. 2016). Within that subclade the resolution is poor, except for a strongly supported group comprising $M$. byssacea, $M$. hedlundii, M. micrococca A and B (sensu Czarnota \& Guzow-Krzemińska 2010), M. viridileprosa and M. xanthonica.

Our new accessions of $M$. nowakii from Romania are identical to two accessions of the same species from Poland (Czarnota \& Guzow-Krzemińska 2010). In two further accessions of the same species, also from Poland, one is resolved as nearly identical to $M$. herbarum sp. nov., and the other as sister to accessions of the same species.

All accessions of $M$. prasina from Poland (Czarnota \& Guzow-Krzemińska 2010) are resolved as a lineage (as 'prasina 1' in Fig. 1) separate to those from Belgium, France and the USA (as 'prasina 2' in Fig. 2); thus two cryptic species might be involved and require further study. Within the $M$. prasina group, our sequence of $M$. byssacea is nearly identical to those referred to that species by Czarnota \& Guzow-Krzemińska (2010), our sequence of $M$. viridileprosa is identical to those of Czarnota \& Guzow-Krzemińska (2010) and finally, our sequences of $M$. micrococca belong to M. micrococca "A" of Czarnota \& Krzemińska (2010). While our sequences of $M$. adnata, $M$. denigrata, $M$. doliiformis, $M$. lignaria var. lignaria, $M$. nitschkeana and $M$. pycnidiophora are almost identical to those retrieved from GenBank, this is not the case for M. peliocarpa and M. stipitata. Indeed, our accession of $M$. peliocarpa from the Netherlands is resolved with strong support as closely related to the recently described M. usneae from Madeira (van den Boom \& Ertz 2014) while the sequence from Norway (Andersen \& Ekman 2005) is quite different (26 substitutions for the mtSSU sequence). The same applies to our 


\begin{tabular}{|c|c|c|c|c|}
\hline Name & Country & $\begin{array}{l}\text { Collector, year, collection number, herbarium } \\
\text { and DNA sample number where appropriate }\end{array}$ & Publication & $\begin{array}{l}\text { GenBank } \\
\text { Acc. no. }\end{array}$ \\
\hline Byssoloma leucoblepharum & Portugal & S. Ekman, -, 3502, BG & Andersen \& Ekman 2005 & AY567778 \\
\hline B. subdiscordans & USA & T. Tønsberg, -, 25968, BG & Andersen \& Ekman 2005 & AY567779 \\
\hline Micarea adnata & Norway & H. L. Andersen, $-, 48, \mathrm{BG}$ & Andersen \& Ekman 2005 & AY567751 \\
\hline M. adnata & France & E. Sérusiaux, 2014, s.n., LG DNA 3438 & & KX459344 \\
\hline M. alabastrites & Norway & H. L. Andersen, -, 17, BG & Andersen \& Ekman 2005 & AY567764 \\
\hline M. assimilata & Sweden & Kanz \& C. Printzen, -, s.n., BG & Andersen \& Ekman 2005 & AY567739 \\
\hline M. botryoides & Norway & H. L. Andersen, -, 79b, BG & Andersen \& Ekman 2005 & AY567741 \\
\hline M. byssacea & Norway & H. L. Andersen, -, 34, BG (as M. micrococca) & Andersen \& Ekman 2005 & AY567749 \\
\hline M. byssacea & Estonia & P. Czarnota $\mathcal{E}$ B. Guzow-Krzemińska, -, GPN 4781 & $\begin{array}{l}\text { Czarnota \& Guzow-Krzemińska } \\
2010\end{array}$ & EF453670 \\
\hline M. byssacea & Estonia & P. Czarnota $\mathcal{E}$ B. Guzow-Krzemińska, -, GPN 3956 & $\begin{array}{l}\text { Czarnota \& Guzow-Krzemińska } \\
2010\end{array}$ & EF453690 \\
\hline M. byssacea & Poland & P. Czarnota \& B. Guzow-Krzemińska, -, GPN 4751 & $\begin{array}{l}\text { Czarnota \& Guzow-Krzemińska } \\
2010\end{array}$ & EF453664 \\
\hline M. byssacea & Germany & $\begin{array}{l}\text { P. van den Boom, 2014, 50037, hb. van den Boom, } \\
\text { LG DNA } 3495\end{array}$ & & KX459345 \\
\hline M. cinerea & Norway & T. Tønsberg, -, 28572, BG & Andersen \& Ekman 2005 & AY567763 \\
\hline M. coppinsii & Norway & T. Tønsberg, -, 26075, BG & Andersen \& Ekman 2005 & AY567761 \\
\hline M. deminuta & Not specified & Z. Palice $\mathcal{E}$ Voriskova, -, 6745, hb. Palice & & AY756446 \\
\hline M. denigrata & Poland & P. Czarnota \& B. Guzow-Krzemińska, -, GPN 4593 & $\begin{array}{l}\text { Czarnota \& Guzow-Krzemińska } \\
\quad 2010\end{array}$ & EF453681 \\
\hline M. denigrata & Netherlands & A. M. Brand, 2014, 63258, hb. Brand, LG DNA 3851 & & KX459346 \\
\hline M. denigrata & Germany & E. Sérusiaux, 2015, s.n., LG DNA 4381 & & KX459347 \\
\hline M. doliiformis & United Kingdom & A. Orange, 2006, s.n., A. Orange, LG, NMW & Sérusiaux et al. 2010 & GU138666 \\
\hline M. doliiformis & Canary Isl., Tenerife & $\begin{array}{l}\text { P. van den Boom, 2014, 52014, hb. van den Boom, } \\
\text { LG DNA } 4239\end{array}$ & & KX459348 \\
\hline M. elachista & Sweden & Koffman, -, 399, hb. Koffman & Andersen \& Ekman 2005 & AY567755 \\
\hline M. elachista & Poland & P. Czarnota \& B. Guzow-Krzemińska, -, GPN 2986 & $\begin{array}{l}\text { Czarnota \& Guzow-Krzemińska } \\
2010\end{array}$ & EF453680 \\
\hline M. erratica & Sweden & U. Arup, -, 99192, hb. Arup & Andersen \& Ekman 2005 & AY567737 \\
\hline M. eximia & Not specified & Hermansson,-, 8866b, UPS & & AY756447 \\
\hline M. flagellispora & Australia, Tasmania & G. Kantvilas,-, 60/90, UPS & & AY756448 \\
\hline M. hedlundii & Poland & P. Czarnota \& B. Guzow-Krzemińska, -, GPN 3895 & $\begin{array}{l}\text { Czarnota \& Guzow-Krzemińska } \\
2010\end{array}$ & EF453672 \\
\hline M. hedlundii & Poland & P. Czarnota \& B. Guzow-Krzemińska, -, GPN 3915 & $\begin{array}{l}\text { Czarnota \& Guzow-Krzemińska } \\
2010\end{array}$ & EF453667 \\
\hline
\end{tabular}




\begin{tabular}{|c|c|c|c|c|}
\hline Name & Country & $\begin{array}{l}\text { Collector, year, collection number, herbarium } \\
\text { and DNA sample number where appropriate }\end{array}$ & Publication & $\begin{array}{l}\text { GenBank } \\
\text { Acc. no. }\end{array}$ \\
\hline Micarea hedlundii & Poland & P. Czarnota Eீ B. Guzow-Krzemińska, -, GPN 4589 & $\begin{array}{l}\text { Czarnota \& Guzow-Krzemińska } \\
2010\end{array}$ & EF453677 \\
\hline M. herbarum sp. nov. Type & Netherlands & $\begin{array}{l}\text { P. E G. van den Boom, 2015, 52575, hb. van den Boom, } \\
\text { LG DNA } 4236\end{array}$ & & KX459349 \\
\hline M. herbarum sp. nov. & Netherlands & A. M. Brand, 2014, 63193, hb. Brand, LG DNA 3852 & & KX459350 \\
\hline M. herbarum sp. nov. & Poland & $\begin{array}{l}\text { P. Czarnota } \mathcal{E} \text { B. Guzow-Krzemińska, -, GPN } 4634 \\
\text { (as M. nowakii) }\end{array}$ & $\begin{array}{l}\text { Czarnota \& Guzow-Krzemińska } \\
2010\end{array}$ & EF453692 \\
\hline M. cf. herbarum & Poland & $\begin{array}{l}\text { P. Czarnota E B. Guzow-Krzemińska, -, GPN } 3464 \\
\text { (as M. nowakii) }\end{array}$ & $\begin{array}{l}\text { Czarnota \& Guzow-Krzemińska } \\
2010\end{array}$ & EF453665 \\
\hline M. incrassata & Not specified & T. Tønsberg, -, $17593(\mathrm{BG})$ & Andersen \& Ekman 2005 & AY756449 \\
\hline M. lapillicola & Czech Republic & C. Printzen, -, s.n., BG & Andersen \& Ekman 2005 & AY567735 \\
\hline M. leprosula & Norway & H. L. Andersen, -, 35, BG & Andersen \& Ekman 2005 & AY567762 \\
\hline M. lignaria var. lignaria & Norway & H. L. Andersen, -, 18, BG & Andersen \& Ekman 2005 & AY567748 \\
\hline M. lignaria var. lignaria & France & E. Sérusiaux, 2014, s.n., LG DNA 3435 & & KX459351 \\
\hline M. lignaria var. lignaria & Romania & E. Sérusiaux, 2015, s.n., LG DNA 4375 & & KX459352 \\
\hline M. lithinella & Norway & H. L. Andersen, -, 80b, BG & Andersen \& Ekman 2005 & AY567734 \\
\hline M. lynceola & Czech Republic & Z. Palice, 1996, -, hb. Palice & Andersen \& Ekman 2005 & AY567738 \\
\hline M. meridionalis sp. nov. Type & Portugal & $\begin{array}{l}\text { P. van den Boom, 2015, -, hb. van den Boom, } \\
\text { LG DNA } 4279\end{array}$ & & KX459353 \\
\hline M. meridionalis sp. nov. & Portugal & $\begin{array}{l}\text { P. van den Boom, 2015, -, hb. van den Boom, } \\
\text { LG DNA } 4281\end{array}$ & & KX459354 \\
\hline M. meridionalis sp. nov. & Portugal & $\begin{array}{l}\text { P. van den Boom, 2015, -, hb. van den Boom, } \\
\text { LG DNA } 4581\end{array}$ & & KX459355 \\
\hline M. marginata & Not specified & S. Bayerová, f. Liska \& Z. Palice,-, 5159, hb. Palice & & AY756451 \\
\hline M. melaena & Norway & H. L. Andersen, -, 25, BG & Andersen \& Ekman 2005 & AY567743 \\
\hline M. micrococca & Poland & P. Czarnota Eீ B. Guzow-Krzemińska, -, GPN 3179 & $\begin{array}{l}\text { Czarnota \& Guzow-Krzemińska } \\
2010\end{array}$ & EF453674 \\
\hline M. micrococca & Estonia & P. Czarnota Eீ B. Guzow-Krzemińska, -, GPN 4782 & $\begin{array}{l}\text { Czarnota \& Guzow-Krzemińska } \\
2010\end{array}$ & EF453676 \\
\hline M. micrococca & Poland & P. Czarnota Eீ B. Guzow-Krzemińska, -, GPN 4179 & $\begin{array}{l}\text { Czarnota \& Guzow-Krzemińska } \\
2010\end{array}$ & EF453691 \\
\hline M. micrococca & Poland & P. Czarnota Eீ B. Guzow-Krzemińska, -, GPN 3632 & $\begin{array}{l}\text { Czarnota \& Guzow-Krzemińska } \\
2010\end{array}$ & EF453668 \\
\hline M. micrococca & Poland & P. Czarnota Eீ B. Guzow-Krzemińska, -, GPN 4553 & $\begin{array}{l}\text { Czarnota \& Guzow-Krzemińska } \\
2010\end{array}$ & EF453683 \\
\hline M. micrococca & Poland & P. Czarnota E̊ B. Guzow-Krzemińska, -, GPN 4059 & $\begin{array}{l}\text { Czarnota \& Guzow-Krzemińska } \\
2010\end{array}$ & EF453663 \\
\hline M. micrococca & Poland & P. Czarnota Eீ B. Guzow-Krzemińska, -, GPN 4456 & $\begin{array}{l}\text { Czarnota \& Guzow-Krzemińska } \\
2010\end{array}$ & EF453662 \\
\hline
\end{tabular}


TABLE 1 (continued).

\begin{tabular}{|c|c|c|c|c|}
\hline Name & Country & $\begin{array}{l}\text { Collector, year, collection number, herbarium } \\
\text { and DNA sample number where appropriate }\end{array}$ & Publication & $\begin{array}{l}\text { GenBank } \\
\text { Acc. no. }\end{array}$ \\
\hline Micarea micrococca & Netherlands & $\begin{array}{l}\text { P. E B. van den Boom, 2014, 50314, hb. van den Boom, } \\
\text { LG DNA } 3853\end{array}$ & & KX459356 \\
\hline M. micrococca & Netherlands & $\begin{array}{l}\text { P. E B B. van den Boom, 2014, 51244, hb. van den Boom, } \\
\text { LG DNA } 3855\end{array}$ & & KX459357 \\
\hline M. micrococca & Netherlands & $\begin{array}{l}\text { P. E B. van den Boom, 2015, 52570, hb. van den Boom, } \\
\text { LG DNA } 4237\end{array}$ & & KX459358 \\
\hline M. misella & Norway & H. L. Andersen, -, 73, BG & Andersen \& Ekman 2005 & AY567752 \\
\hline M. misella & Poland & P. Czarnota \& B. Guzow-Krzemińska, -, GPN 4593 & $\begin{array}{l}\text { Czarnota \& Guzow-Krzemińska } \\
\quad 2010\end{array}$ & EF453687 \\
\hline M. myriocarpa & Norway & H. L. Andersen, -, 37, BG & Andersen \& Ekman 2005 & AY567736 \\
\hline M. nitschkeana & Czech Republic & C. Printzen, -, s.n., BG & Andersen \& Ekman 2005 & AY567758 \\
\hline M. nitschkeana & Poland & P. Czarnota \& B. Guzow-Krzemińska, -, GPN 3306 & $\begin{array}{l}\text { Czarnota \& Guzow-Krzemińska } \\
\quad 2010\end{array}$ & EF453685 \\
\hline M. nowakii & Poland & P. Czarnota $\mathcal{E}$ B. Guzow-Krzemińska, -, GPN 4181 & $\begin{array}{l}\text { Czarnota \& Guzow-Krzemińska } \\
2010\end{array}$ & EF453688 \\
\hline M. nowakii & Poland & P. Czarnota $\mathcal{E}$ B. Guzow-Krzemińska, -, GPN 4688 & $\begin{array}{l}\text { Czarnota \& Guzow-Krzemińska } \\
2010\end{array}$ & EF453689 \\
\hline M. nowakii & Romania & E. Sérusiaux, 2015, s.n., LG DNA 4385 & & KX459360 \\
\hline M. paratropa & Norway & H. L. Andersen, -, 94, BG & Andersen \& Ekman 2005 & AY567740 \\
\hline M. peliocarpa & Norway & H. L. Andersen, -, 29, BG & Andersen \& Ekman 2005 & AY567760 \\
\hline M. peliocarpa & Netherlands & $\begin{array}{l}\text { P. van den Boom, 2014, 51318, hb. van den Boom, } \\
\text { LG DNA } 3847\end{array}$ & & KX459361 \\
\hline M. prasina & USA & T. Tønsberg, -, 30856, BG & $\begin{array}{l}\text { Czarnota \& Guzow-Krzemińska } \\
2010\end{array}$ & AY756452 \\
\hline M. prasina & France & E. Sérusiaux, 2014, s.n., LG DNA 3437 & & KX459362 \\
\hline M. prasina & Belgium & E. Sérusiaux, 2014, s.n., LG DNA 3609 & & KX459363 \\
\hline M. prasina & Poland & P. Czarnota E B. Guzow-Krzemińska, -, GPN 3913 & $\begin{array}{l}\text { Czarnota \& Guzow-Krzemińska } \\
\quad 2010\end{array}$ & EF453675 \\
\hline M. prasina & Poland & P. Czarnota $\mathcal{E}$ B. Guzow-Krzemińska, -, GPN 3914 & $\begin{array}{l}\text { Czarnota \& Guzow-Krzemińska } \\
\quad 2010\end{array}$ & EF453669 \\
\hline M. prasina & Poland & P. Czarnota \& B. Guzow-Krzemińska, -, GPN 4319 & $\begin{array}{l}\text { Czarnota \& Guzow-Krzemińska } \\
2010\end{array}$ & EF453679 \\
\hline M. prasina & Poland & P. Czarnota \& B. Guzow-Krzemińska, -, GPN 4489 & $\begin{array}{l}\text { Czarnota \& Guzow-Krzemińska } \\
2010\end{array}$ & EF453678 \\
\hline M. prasinella & USA & B. McCune, -, 35337, BG & Andersen \& Ekman 2005 & AY567745 \\
\hline M. pycnidiophora & USA & T. Tønsberg, -, 30881, BG & Andersen \& Ekman 2005 & AY567754 \\
\hline
\end{tabular}




\begin{tabular}{|c|c|c|c|c|}
\hline Name & Country & $\begin{array}{l}\text { Collector, year, collection number, herbarium } \\
\text { and DNA sample number where appropriate }\end{array}$ & Publication & $\begin{array}{l}\text { GenBank } \\
\text { Acc. no. }\end{array}$ \\
\hline Micarea soralifera & Poland & M. Kukwa, 2014, 12722, UGDA & Guzow-Krzemińska et al. 2016 & KT119884 \\
\hline M. soralifera & Poland & M. Kukwa, 2014, 12999, UGDA & Guzow-Krzemińska et al. 2016 & KT119885 \\
\hline M. soralifera Type & Poland & M. Kukwa $13001 \&$ A. Eubek, 2014, UGDA & Guzow-Krzemińska et al. 2016 & KT119886 \\
\hline M. stipitata & USA & S. Ekman, -, s.n., BG & Andersen \& Ekman 2005 & AY567756 \\
\hline M. stipitata & Canary Is., Tenerife & E. Sérusiaux, 2014, s.n., LG DNA 3816 & & KX459365 \\
\hline M. subviridescens & U.K., Scotland & P. Czarnota $\mathcal{G}$ B. Guzow-Krzemińska, -, GPN 3599 & $\begin{array}{l}\text { Czarnota \& Guzow-Krzemińska } \\
2010\end{array}$ & EF453666 \\
\hline M. synotheoides & Norway & H. L. Andersen, -, 47, BG & Andersen \& Ekman 2005 & AY567756 \\
\hline M. tomentosa & Poland & P. Czarnota \& B. Guzow-Krzemińska, -, GPN 3949 & $\begin{array}{l}\text { Czarnota \& Guzow-Krzemińska } \\
2010\end{array}$ & EF453686 \\
\hline M. turfosa & Norway & H. L. Andersen, -, 59, BG & Andersen \& Ekman 2005 & AY567742 \\
\hline M. usneae & Portugal, Madeira & P.E B. van den Boom, 2012, 48057, BR & van den Boom \& Ertz 2014 & KF569511 \\
\hline M. viridileprosa & Netherlands & $\begin{array}{l}\text { P. E B. van den Boom, 2013, 50066, hb. van den Boom, } \\
\text { LG DNA } 3493\end{array}$ & & KX459366 \\
\hline M. viridileprosa & Poland & P. Czarnota \& B. Guzow-Krzemińska, -, GPN 3436 & $\begin{array}{l}\text { Czarnota \& Guzow-Krzemińska } \\
2010\end{array}$ & EF453671 \\
\hline M. viridileprosa & Poland & P. Czarnota $\mathcal{E}$ B. Guzozw-Krzemińska, -, GPN 3869 & $\begin{array}{l}\text { Czarnota \& Guzow-Krzemińska } \\
2010\end{array}$ & EF453673 \\
\hline M. viridileprosa & Poland & P. Czarnota E B. Guzow-Krzemińska, -, GPN 4518 & $\begin{array}{l}\text { Czarnota \& Guzow-Krzemińska } \\
2010\end{array}$ & EF453684 \\
\hline M. viridileprosa & Poland & P. Czarnota Eீ B. Guzow-Krzemińska, -, GPN 4527 & $\begin{array}{l}\text { Czarnota \& Guzow-Krzemińska } \\
2010\end{array}$ & EF453682 \\
\hline M. xanthonica & USA & T. Tonsberg, -, 25674, BG & $\begin{array}{l}\text { Czarnota \& Guzow-Krzemińska } \\
2010\end{array}$ & AY756454 \\
\hline Szczawinskia leucopoda & USA & T. Tønsberg, -, 30044, BG & Andersen \& Ekman 2005 & AY567746 \\
\hline
\end{tabular}




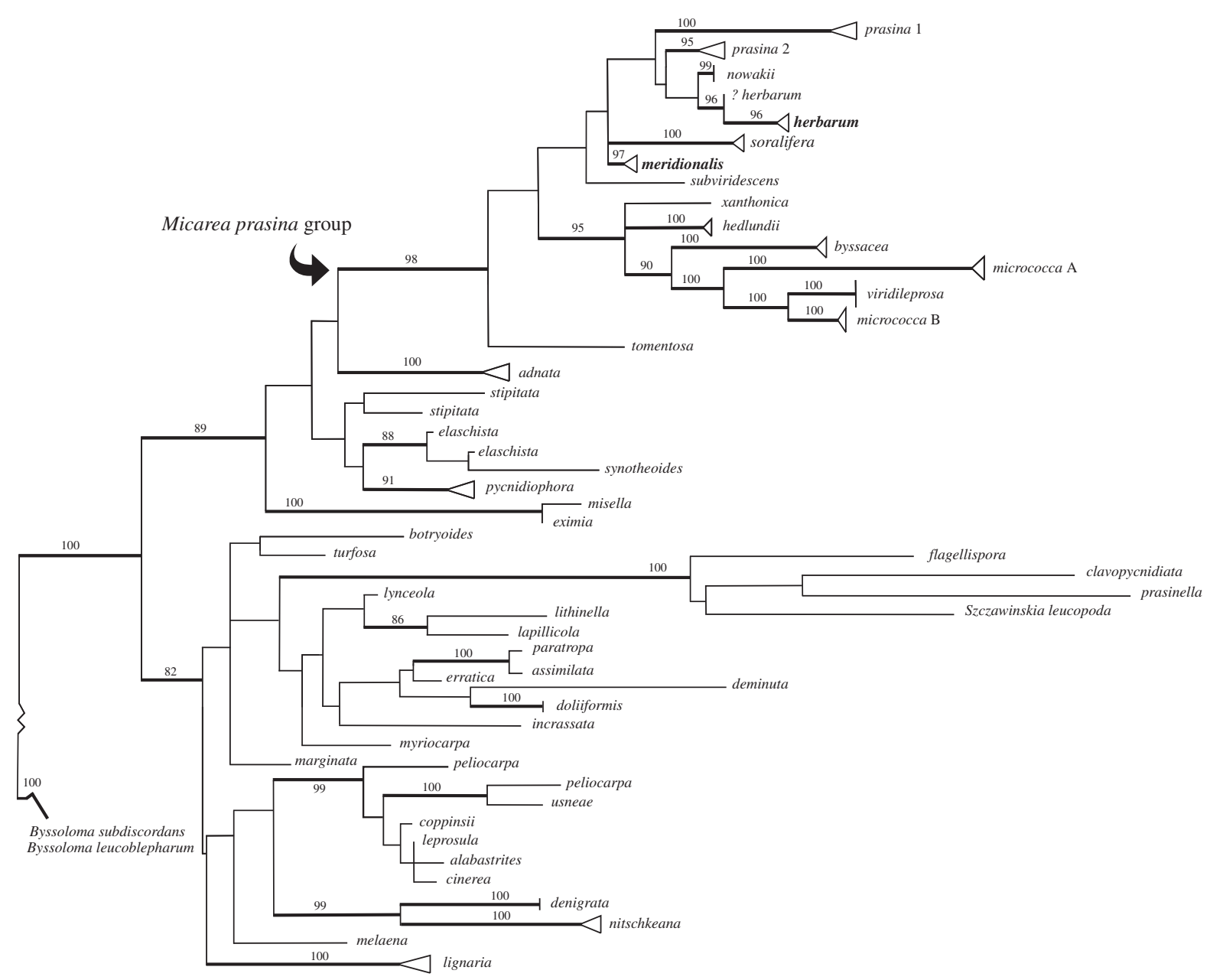

FIG. 1. Most-likely phylogenetic tree for species of Micarea obtained from mtSSU sequences. Branches in bold are those that obtained ML bootstrap support $>70 \%$ and are indicated above branches. New species are in bold. 
sequences for $M$. stipitata from Tenerife (Canary Islands) which differ by 22 substitutions from the sequence from the USA retrieved from GenBank (Andersen \& Ekman 2005). Thus, our limited sampling illuminates the rather poorly known diversity within Micarea.

Morphological, anatomical and chemical results are included in the description of both new species.

\section{Taxonomy}

\section{Micarea herbarum Brand, Coppins, Sérus. \& van den Boom sp. nov.}

\author{
MycoBank No.: MB 811051
}

\begin{abstract}
Species inconspicuous, with a very thin thallus comprised of small greenish, flattened or slightly convex areoles, and often covered by a thin film of gelatinous green algal cells. Apothecia abundant, dark brown to black, 0.15-0.25 mm diam., immarginate. Ascospores ellipsoid, 6.5-9.7 $\times 2.0-2.6 \mu \mathrm{m},(0-) 1$-septate. Pigment Sedifolia-grey, $\mathrm{K}+$ violet. Mesopycnidia often abundant; mesoconidia shortly bacilliform, 3.8-6.1 × 1.0-1.2(-1.3) $\mu \mathrm{m}$. No secondary compounds detected.

Type: the Netherlands, Noord-Brabant, S of Oirschot, S rim of Oirschotse Heide, Pinus-Quercus forest with many fallen trunks of Quercus, on wood of fallen trunk, TDN grid ref. 51.33.42, 22 January 2015, P. E B. van den Boom 52575 (LG-holotype; hb. v.d. Boom-isotype).
\end{abstract}

(Fig. 2A-D)

Thallus very thin, consisting of small greenish, flattened or slightly convex areoles, less than $0.1 \mathrm{~mm}$ diam., with crystals, often partly coated by a thin gelatinous film of green algal cells. Photobiont micareoid, 6-8 $\mu \mathrm{m}$ diam., thin-walled, clustered in compact masses.

Apothecia abundant, subglobose, immarginate, 0.15 to $0.25 \mathrm{~mm}$ diam., dark brown to black. Hymenium c. $30-40 \mu \mathrm{m}$ high; epihymenium with dark green-brown (K+ violet) spots; paraphyses sparse, branched, c. $1 \cdot 2-1.4 \mu \mathrm{m}$ wide. Hypothecium hyaline. Asci 20-28 $\times 7-12 \mu \mathrm{m}$. Ascospores ellipsoid, 6.5-9.7 × 2.0-2.6 $\mu \mathrm{m},(0-) 1$-septate.

Mesopycnidia often abundant, c. 40-80 $\mu \mathrm{m}$, top dark greenish grey ( $\mathrm{K}+$ violet), formed inside a thallus granule, or outside of the lichenized thallus in the gelatinous matrix of free algal cells. Mesoconidia shortly bacilliform, rarely obovoid, 3.8-6.1 × 1.0-1.2 $(-1 \cdot 3) \mu \mathrm{m}$, non-septate.

Chemistry. No chemical compounds; pigment Sedifolia-grey (Meyer \& Printzen 2000), $\mathrm{K}+$ violet.

Etymology. The epithet chosen for this species refers to a quite unusual habitat (decaying herbs) on which it has been found several times.

Habitat and distribution. On soft and decaying wood, on standing dead trunks, on dead and wet stems of herbaceous plants, or directly on soil. In the Netherlands, accompanying species on decaying wood include Absconditella sp., Micarea micrococca and Placynthiella dasaea, and on soil Absconditella fossarum and Thelocarpon lichenicola. It is distributed throughout the Netherlands, and has been detected in one collection from Poland, filed under the recently described $M$. nowakii (Czarnota 2007). In the latter collection, it grows on decorticated wood with $M$. denigrata and $M$. misella. The species is very inconspicuous, and can be easily overlooked because it is frequently covered with gelatinous algal cells. No doubt it will be found in other European countries.

Notes. In the monograph and revisions of the genus in Europe (Coppins 1983, 2009; Czarnota 2007), this new species would key out as Micarea denigrata if no chemical test for gyrophoric acid is performed. Indeed, it looks like a small or depauperate $M$. denigrata, a common and highly polymorphic species, but clearly differs by the lack of gyrophoric acid and the shorter mesoconidia in $M$. denigrata $(3 \cdot 0-4 \cdot 2(-5 \cdot 0) \times 1 \cdot 4-1 \cdot 8(-2 \cdot 0) \mu \mathrm{m}$, fide Czarnota 2007). We interpret the conidia produced by our new species as mesoconidia as they originate in rather large pycnidia and they are regularly bacilliform (rarely obovoid) in shape, and thus akin to the mesoconidia produced by $M$. denigrata. No validly published epithet reduced into synonymy with this species in Coppins (1983) can be assigned to the new species. Micarea misella might also be confused with this species and differs mainly by its simple ascospores. 

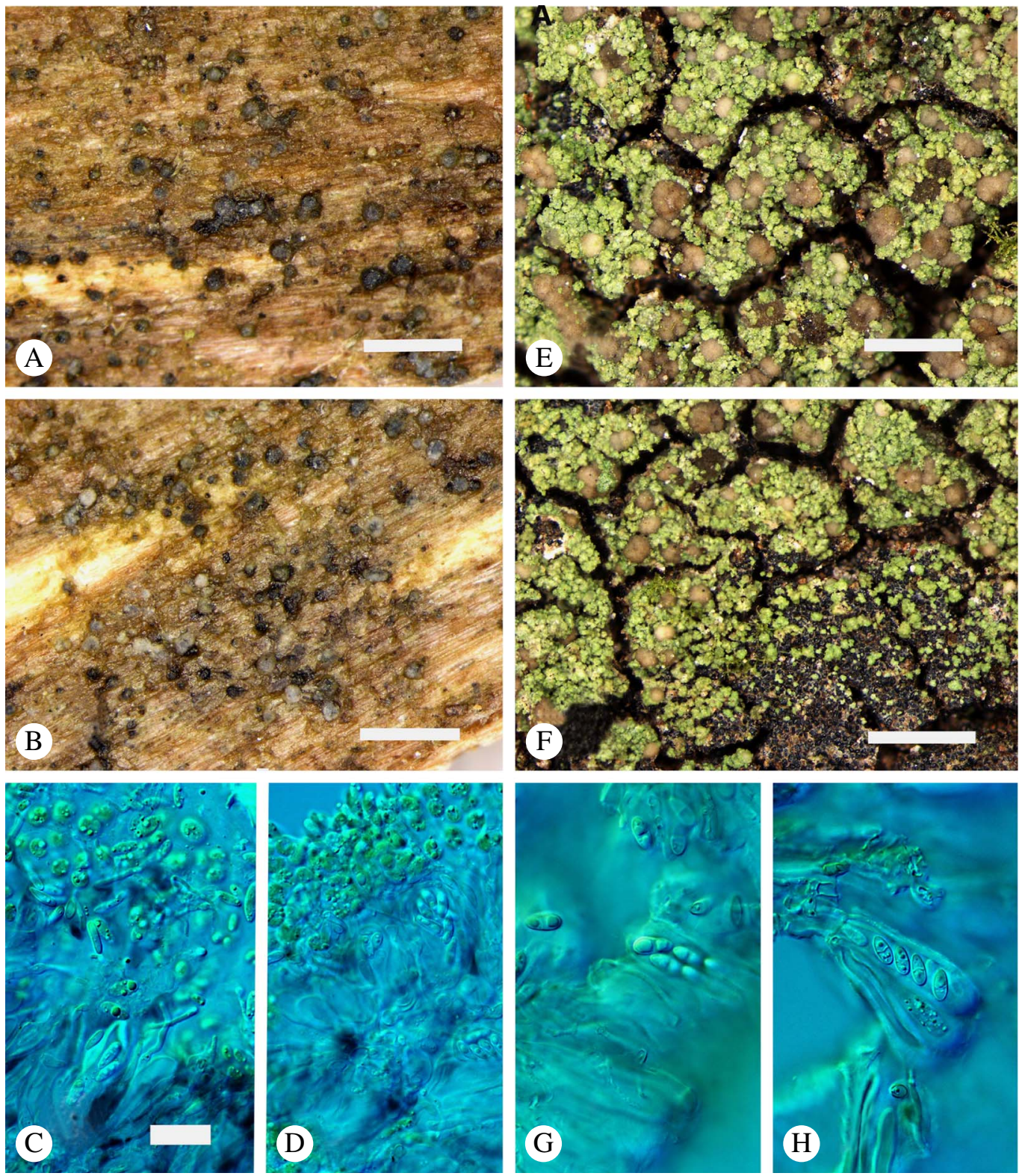

Fig. 2. Micarea herbarum and M. meridionalis. A-D, Micarea herbarum (holotype); A \& B, habitus; C \& D, ascospores with green algal cells growing over the hymenium. E-H, Micarea meridionalis (holotype); E \& F, habitus; G \& H, ascospores. Scales: A, B, E \& F $=0.5 \mathrm{~mm}$; C, D, G \& H $=20 \mu \mathrm{m}$. In colour online.

In addition, $M$. denigrata and $M$. misella are resolved outside the $M$. prasina clade in which $M$. herbarum is nested with strong support. Phylogenetic inferences from $\mathrm{mtSSU}$ sequences position $M$. herbarum in an unsupported clade within the strongly supported $M$. prasina group, together with both lineages referred to as $M$. prasina and $M$. nowakii, a species described from Poland (Czarnota 2007). $M$. nowakii was segregated from $M$. denigrata 
and M. misella based on the production of micareic acid (vs gyrophoric acid or no substances for the other two, respectively). Compared with $M$. herbarum, $M$. nowakii has slightly smaller ascospores $(0(-1)$-septate, $6 \cdot 0-8 \cdot 0(-8 \cdot 5) \times 2 \cdot 0-3 \cdot 0(-3 \cdot 2) \mu \mathrm{m}$ fide Czarnota 2007) and shorter and wider mesoconidia (3.5-4.0 × 1.5-1.8 $\mu \mathrm{m}$ fide Czarnota 2007).

The position of the two accessions of $M$. herbarum in our phylogenetic tree points to a relationship between $M$. herbarum and $M$. nowakii, or even that $M$. herbarum cannot be distinguished from $M$. nowakii. Among the mtSSU sequences published by Czarnota \& Guzow-Krzemińska (2010) for $M$. nowakii, one accession (Poland, P. Czarnota 4634, GPN) is resolved with strong support as closely related to $M$. herbarum. We found that this collection represents $M$. herbarum (no micareic acid detected by TLC); a further collection (Poland, P. Czarnota 3464, GPN) might represent either a further species in that group, or a variant of $M$. herbarum. In fact, this collection does not have apothecia but only pycnidia; no crystals of any lichen substance could be detected by microcrystallization and the phylogenetic tree resolved it as sister with all accessions referred to $M$. herbarum.

Micarea melanobola (Nyl.) Coppins is a species so far known only from the type collection in Finland, collected in 1866 (Coppins 1983). Although it has been reduced into synonymy with $M$. prasina by Czarnota (2007), we suspect this species to be close to $M$. nowakii and thus to M. herbarum. Indeed, M. melanobola has paraphyses with "apices thickened with greenish $(\mathrm{K}+$ violet) pigment and up to $1.7 \mu \mathrm{m}$ wide overtopping the tops of asci" (Coppins 1983), a typical feature that seems to be quite similar to the paraphyses of $M$. nowakii described with "apices, thickened to $2-2.5 \mu \mathrm{m}$ and dull olive pigment", said to react $\mathrm{K}+$ violet (legend to fig. 46 in Czarnota 2007). The chemistry of the type of M. melanobola could not be studied for lack of material (Coppins 1983). The genuine identity of the type material of $M$. melanobola should therefore be re-evaluated.

It is worth mentioning that $M$. herbarum is the first species within the prasina group that does not produce any secondary compounds other than the pigment present in apothecia.

The biology of $M$. herbarum sp. nov. is remarkable, as it very often grows over or within a gelatinous film of green algal cells. Hyphae connected with the apothecia and pycnidia penetrate into this layer. Furthermore, these unknown algae can penetrate into the subhymenium of the Micarea apothecia.

Specimens examined. The Netherlands: Groningen: $1.8 \mathrm{~km} \mathrm{~N}$ van Sellingen, E bank of Ruiten Aa, N of bridge near Rijsdam, $52^{\circ} 57 \cdot 8^{\prime} \mathrm{N}, 7^{\circ} 8.6^{\prime} \mathrm{E}$, open area, loamy soil recently scraped, 1999, M. Brand 39768 (hb. Brand). Overijssel: Hardenberg, Rheezerveen, Klimberg, $52^{\circ} 34.8 ' \mathrm{~N}, 6^{\circ} 32.9^{\prime} \mathrm{E}$, dead wood in forest, 2010 , M. Brand 61133 (hb. Brand). Noord-Holland: Santpoort, Duin en Kruidberg, tank ditch between Argus and Westerveld, $52^{\circ} 26 \cdot 6^{\prime} \mathrm{N}, 4^{\circ} 37 \cdot 4^{\prime} \mathrm{E}$, open forest in dune area, wood of Populus alba branches, 2014, M. Brand 63193 (hb. Brand). Zuid-Holland: Zoetermeer, Sprinterpad, N of Westerpark, $52^{\circ} 3.6^{\prime} \mathrm{N}, 4^{\circ} 26 \cdot 7^{\prime} \mathrm{E}$, rotting wood of dead Populus trunks, 2010, M. Brand 60950 (hb. Brand). Gelderland: Vierhouten, Elspeetsche Heide, W of bicycle trail, $52^{\circ} 18.6^{\prime} \mathrm{N}, 5^{\circ} 48.5^{\prime} \mathrm{E}$, rotting wood in heathland, 1999 , M. Brand 39206 (hb. Brand); Heerde, Sprengen, $52^{\circ} 23.9^{\prime} \mathrm{N}, 6^{\circ} 0 \cdot 2^{\prime} \mathrm{E}$, wood of bridge in forest, 1973 , M. Brand 3254 (hb. Brand); Garderen, Speulderbos, $14 \mathrm{~m} \mathrm{E}$ of Dodenweg, $52^{\circ} 14.65^{\prime} \mathrm{N}, 5^{\circ} 41.53^{\prime} \mathrm{E}$, dead wood of fallen Fagus in forest, 1998, M. Brand 37726 (hb. Brand). Zeeland: Tholen, recently reclaimed area between Slaakdam and Haaftenpolder, S side, $51^{\circ} 36 \cdot 3^{\prime} \mathrm{N}$, $4^{\circ} 10 \cdot 8^{\prime} \mathrm{E}$, twigs in open grass vegetation, 1983, M. Brand 33842 (hb. Brand). Noord-Brabant: E of Oirschot, Oirschotse Heide, N side of secondary road to Oirschot, Pinus forest and edge of Calluna heathland with some Quercus robur trees, $51^{\circ} 30^{\prime} \mathrm{N}, 5^{\circ} 21^{\prime} \mathrm{E}$, wood of fallen trunk, 2014, P. E B. van den Boom 52533 (hb. v.d. Boom). Limburg: Weert, Kolenhofweg, $1 \mathrm{~km} \mathrm{NE}$ of Mildert, $51^{\circ} 14.6 \mathrm{~N}, 5^{\circ} 48.3^{\prime} \mathrm{E}$, rotting wood of trunk in young Pinus plantation, 2000, M. Brand 41201 (hb. Brand); Swalmen, $2 \mathrm{~km}$ NE, Boschheide, $51^{\circ} 14 \cdot 4^{\prime} \mathrm{N}, 6^{\circ} 3 \cdot 7^{\prime} \mathrm{E}$, recently partly scraped Calluna heathland, 1992, M. Brand 29016 (hb. Brand); E of Wanssum, NE of Wellerlooi, De Hamert, open Pinus forest with Calluna heathland, a few scattered Pinus strobus trees and fallen trunks, $51^{\circ} 32 \cdot 7^{\prime} \mathrm{N}, 6^{\circ} 08 \cdot 6^{\prime} \mathrm{E}$, dead wood of fallen P. strobus, 2015 , P. E B. van den Boom 53197 (hb. v.d. Boom).-Poland: Pojezierze Lubuskie Lakeland, S of Motski Village, $52^{\circ} 14^{\prime} 42^{\prime \prime} \mathrm{N}, 15^{\circ} 22^{\prime} 14^{\prime \prime} \mathrm{E}$, on wooden fence in well-lit place near the edge of pine forest, 2005, P. Czarnota 4634 (GPN).

\section{Micarea meridionalis van den Boom, Brand, Coppins \& Sérus. sp. nov.}

\section{MycoBank No.: MB 811050}

Thallus areolate, areoles subglobose to irregularly flattened, 40-100 $\mu \mathrm{m}$ diam., pale to medium greenish, 
or greenish grey to pale brownish; apothecia abundant, $0 \cdot 10-0 \cdot 30(-0 \cdot 35) \mathrm{mm}$ diam., pale to dark brownish grey; ascospores ellipsoid, 1(-3)-septate, 8.0-9.4 × 3.4-4.0 $\mu \mathrm{m}$; mesopycnidia often present, $50-70 \mu \mathrm{m}$ diam.; mesoconidia $5.8-6.7 \times 1.0-1.2 \mu \mathrm{m}$, bacilliform to fusiform. Thallus and apothecia with micareic acid.

Type: Portugal, Alentejo, ESE of Odemira, c. $4 \mathrm{~km} \mathrm{E}$ of Santa Clara-a-Velha, near storage lake (W side), Pinus forest, $37^{\circ} 30 \cdot 90^{\prime} \mathrm{N}, 8^{\circ} 26 \cdot 66^{\prime} \mathrm{W}, 150 \mathrm{~m}, 16$ February 2015 , on Pinus, P. \& B. van den Boom 52904 (LG-holotype; hb. Brand, hb. v.d. Boom-isotypes).

\section{(Fig. 2E-H)}

Thallus \pm effuse, up to $4 \mathrm{~cm}$ wide, appearing granular, with ecorticate areoles, $40-100 \mu \mathrm{m}$ diam., subglobose to irregularly flattened, coalescing and forming a continuous crust or scattered patches; upper surface usually smooth, pale to medium greenish, or greenish grey to brownish grey to pale brown, matt to slightly shiny, up to $20 \mu \mathrm{m}$ thick, mostly thinly coated by gelatinous algae, fine crystals present; rarely forming soredioid structures consisting of loose goniocysts $10-14 \mu \mathrm{m}$ in diam. Photobiont micareoid, algal cells globose, 4-6 $\mu \mathrm{m}$ diam., thin-walled, clustered in compact masses.

Apothecia 0.10-0.30(-0.35) $\mathrm{mm}$ diam., abundant, scattered, sometimes tuberculate, immersed to semi-immersed in thallus granules and thus seemingly marginate, because of thallus parts present on the edge of apothecium, eventually becoming immarginate; disc beige or pale to dark brownish grey, never black, slightly to moderately convex, a few crystals sometimes seen in apothecium section. Hymenium hyaline, 40-50 $\mu \mathrm{m}$ high, with spots pale greyish brown, $\mathrm{K}+$ violet, $\mathrm{N}+$ reddish; excipulum in young apothecia hardly distinct, up to $10 \mu \mathrm{m}$ wide; paraphyses abundantly branched, c. $1.2 \mu \mathrm{m}$ wide, tips not or sometimes slightly widened, up to $1.5 \mu \mathrm{m}$, not pigmented; epithecium olive-greenish, $\mathrm{K}+$ violet, $\mathrm{N}+$ reddish; hypothecium hyaline. Asci slightly clavate, $35-40 \times 8-10 \mu \mathrm{m}$, 8-spored. Ascospores ellipsoid, 1(-3)-septate, 8.0-9.4 × 3.4-4.0 $\mu \mathrm{m}$.

Macropycnidia very rare, c. $50 \mu \mathrm{m}$ diam., hyaline but top greyish; macroconidia 15-17 × 1.1-1.3 $\mu \mathrm{m}, 1$-septate, slightly curved. Mesopycnidia often present, immersed, 50-70 $\mu \mathrm{m}$ diam., hyaline; mesoconidia bacilliform to fusiform, $5.8-6.7 \times 1 \cdot 0-1.2 \mu \mathrm{m}$.
Chemistry. Thallus and apothecia $\mathrm{K}-, \mathrm{C}-$, $\mathrm{KC}-, \mathrm{P}-$; micareic acid detected by TLC in thallus and apothecia; pigment Sedifolia-grey (Meyer \& Printzen 2000), K+ violet.

Etymology. The epithet chosen for this species refers to its southern distribution in Europe.

Habitat and distribution. Micarea meridionalis is known from several localities in western Portugal, in lowland and maritime areas, in the Alentejo, Estremadura and Lisboa provinces, between $39^{\circ} 40^{\prime} \mathrm{N}$ and $37^{\circ} 30^{\prime} \mathrm{N}$. In these localities it is a corticolous species, in ruderal and even dusty environments including parklands and roadsides; it has been found on indigenous or planted tree species such as Acacia longifolia, Eucalyptus, Pinus, Morus, Nerium oleander and Thuja. On Acacia longifolia, accompanying species include Arthonia pruinata, Cliostomum griffithii, Coenogonium tavaresiana, Hyperphyscia adglutinata, Lecania naegelii and Lecanora lividocinerea. Diploicia canescens, Waynea stoechadeana, Candelariella reflexa and Physconia grisea are further associated species found growing together. Two further collections have also been found from Calabria/Italy: here the species grows on Pinus trees at sea level in unknown ecological conditions. Further north it is known from the Botanical Garden of Rome in the city centre on Acanthosyris spinescens and Quercus pubescens, and within the urban area of Rome on Quercus suber in a small remnant of semi-natural forest.

Notes. Micarea meridionalis is resolved within the $M$. prasina clade with strong support but its relationships within it are unclear. It is easily distinguished by its granular thallus and the production of micareic acid. The only other Micarea species that produce micareic acid are $M$. nowakii, $M$. prasina s. str. and M. soralifera (Czarnota 2007; Czarnota \& Guzow-Krzemińska 2010; Guzow-Krzemińska et al. 2016). In addition to the typical granular thallus, $M$. meridionalis can be distinguished from $M$. nowakii by its longer mesoconidia (3.5-4.0 $\times 1.5-1.8 \mu \mathrm{m}$ for M. nowakii, fide Czarnota (2007)) and from $M$. prasina s. str. by its shorter 
ascospores $\quad((6-) 8-12(-14) \times(2 \cdot 5-) 3 \cdot 0-4 \cdot 0$ $(-5.5) \mu \mathrm{m}$ for $M$. prasina s. str., fide Czarnota (2007)). Micarea soralifera has similar granular areoles to $M$. meridionalis, but is distinguished by its distinct, mostly discrete soralia. Furthermore, the ecology of $M$. meridionalis is much more ruderal and eutrophic than $M$. nowakii, $M$. prasina s. str. and $M$. soralifera, all of which prefer more acidic and nutrient-poor substrata.

No validly published epithet reduced into synonymy with $M$. prasina in the monograph and revisions of the genus in Europe (Coppins 1983, 2009; Czarnota 2007) can be assigned to the new species.

Specimens examined. Portugal: Estremadura: E of Caldas da Rainha, road to Santa Catarina, between Cabeça Alta and Portela, Pinus forest along road, on Pinus, 2001, P. E B. van den Boom 27727, 27731, 27736 (hb. v.d. Boom). Beira: Nazaré, NE of town, Pinus forest, on Pinus, 2003, M. Brand 49616 (hb. Brand). Setubal: Serra da Arrábida, $2 \mathrm{~km} \mathrm{SW}$ of Aldeia, near gate of former chapel, on old Morus, 2003, M. Brand 49832 (hb. Brand); ibid., on Nerium oleander, M. Brand 49839 (hb. Brand). Alentejo: NE of Cercal, road N261 from Alvalade to São Domingo, mature Quercus suber trees along field, on $Q$. suber, $37^{\circ} 55.91 ' \mathrm{~N}, 8^{\circ} 27.93^{\prime} \mathrm{W}, 2015$, P. E B. van den Boom 53059 (hb. v.d. Boom); WNW of Cercal, N of Vila Nova de Milfontes, Praia da Ilha, coastal outcrops with shrubs, including Acacia longifolia, on Acacia, $37^{\circ} 49 \cdot 79^{\prime} \mathrm{N}, 8^{\circ} 47 \cdot 48^{\prime} \mathrm{W}, 2015, P$. E B. van den Boom 52966 (hb. v.d. Boom); ENE of Cercal, c. $1 \mathrm{~km}$ E of the city, along road N262, rows of mature Eucalyptus globulus trees, on Eucalyptus, $37^{\circ} 48.36^{\prime} \mathrm{N}, 8^{\circ} 38 \cdot 32^{\prime} \mathrm{W}$, 2015, P. E B. van den Boom 53089 (hb. v.d. Boom); WNW of Odemira, E of Almograve, Longueira, roadside Acacia longifolia shrubs, on Acacia, $37^{\circ} 39.41^{\prime} \mathrm{N}$, $8^{\circ} 46 \cdot 17^{\prime} \mathrm{W}, 2015$, P. E B. van den Boom 52664 (hb. v.d. Boom); WNW of Odemira, Cabo Sardão, Acacia longifolia shrubs in coastal area, near lighthouse, on Acacia, $37^{\circ} 35.95^{\prime} \mathrm{N}, 8^{\circ} 48.95^{\prime} \mathrm{W}, 2015, P$. E B. van den Boom 52690 (hb. v.d. Boom); SW of Odemira, Boavista dos Pinheiros, botanical garden 'Parque das Águas', mixed trees, including Salix and Mimosaceae, on a stump, 37 $34.97^{\prime} \mathrm{N}, 8^{\circ} 39.62^{\prime} \mathrm{W}, 2015, P$. E B. van den Boom 52920 (hb. v.d. Boom); SW of Odemira, just $S$ of Zambujeira, $37^{\circ} 31 \cdot 18^{\prime} \mathrm{N}, 8^{\circ} 47 \cdot 11^{\prime} \mathrm{W}$, coastal dune area with Acacia longifolia, on Acacia, 2015, P. E B. van den Boom 53032 (hb. v.d. Boom). Lisboa: Sintra, park, on trunk of Thuja, 2015, P. E B. van den Boom 53940 (hb. v.d. Boom).--Italy: Calabria: Nicotera, on Pinus bark, 2000, D. Puntillo (E). Lazio: Rome, Orto Botanico di Roma, alt. $50 \mathrm{~m}$, on Acanthosyris spinescens, 1999, S. Ravera 3228 (RO); ibid., alt. $60 \mathrm{~m}$, on Quercus pubescens, 1999, G. Brezzi [Ravera 3229] (RO); Rome, Parco di Monte Mario, alt. $137 \mathrm{~m}$, on Quercus suber, 2000, G. Brezzi [Ravera 3231] (RO).
We warmly thank Ido Cremasco and Laurent Gohy for technical assistance in the molecular laboratory and herbarium at the University of Liège, and Paweł Czarnota, Beta Guzow-Krzemińska, Domenico Puntillo and Sonia Ravera for the loan of material and/or providing us with interesting data.

\section{REFERENCES}

Andersen, H. L. \& Ekman, S. (2005) Disintegration of the Micareaceae (lichenized Ascomycota): a molecular phylogeny based on mitochondrial rDNA sequences. Mycological Research 109: 21-30.

Aptroot, A. \& Cáceres, M. E. S. (2014) New lichen species from termite nests in rainforest in Brazilian Rondônia and adjacent Amazonas. Lichenologist 46: 365-372.

Barton, J. \& Lendemer, J. (2014) Micarea micrococca and M. prasina, the first assessment of two very similar species in eastern North America. Bryologist 117: 223-231.

Brand, A. M., van den Boom, P. P. G. \& Sérusiaux, E. (2014) Unveiling a surprising diversity in the lichen genus Micarea (Pilocarpaceae) in Réunion (Mascarenes archipelago, Indian Ocean). Lichenologist 46: 413-439.

Cáceres, M. E. S., Mota, D. A., de Jesus, L. S. \& Aptroot, A. (2013) The new lichen species Micarea corallothallina from Serra da Jibóia, an Atlantic rainforest enclave in Bahia, NE Brazil. Lichenologist 45: 371-373.

Castresana, J. (2000) Selection of conserved blocks from multiple alignments for their use in phylogenetic analysis. Molecular Biology and Evolution 17: 540-552.

Coppins, B. J. (1983) A taxonomic study of the lichen genus Micarea in Europe. Bulletin of the British Museum (Natural History), Botany Series 11: 17-214.

Coppins, B. J. (2009) Micarea. In The Lichens of Great Britain and Ireland (C. W. Smith, A. Aptroot, B. J. Coppins, A. Fletcher, O. L. Gilbert, P. W. James \& P. A. Wolseley, eds): 583-606. London: British Lichen Society.

Córdova-Chávez, O., Aptroot, A., Castillo-Camposa, G., Cáceres, M. E. S. \& Pérez-Pérez, R. E. (2014) Three new lichen species from cloud forest in Veracuz, Mexico. Cryptogamie, Mycologie 35: 157-162.

Cubero, O. F., Crespo, A., Fatehi, J. \& Bridge, P. D. (1999) DNA extraction and PCR amplification method suitable for fresh, herbarium-stored, lichenized, and other fungi. Plant Systematics and Evolution 216: 243-249.

Czarnota, P. (2007) The lichen genus Micarea (Lecanorales, Ascomycota) in Poland. Polish Botanical Studies 23: 1-199.

Czarnota, P. \& Guzow-Krzemińska, B. (2010) A phylogenetic study of the Micarea prasina group shows that Micarea micrococca includes three distinct lineages. Lichenologist 42: 7-21.

Guzow-Krzemińska, B., Czarnota, P., Łubek, A. \& Kukwa, M. (2016) Micarea soralifera sp. nov., a new sorediate species in the $M$. prasina group. Lichenologist 48: 161-169. 
Huneck, S. \& Yoshimura, I. (1996) Identification of Lichen Substances. Berlin, Heidelberg: Springer-Verlag.

Katoh, K. \& Standley, D. M. (2013) MAFFT multiple sequence alignment software version 7: improvements in performance and usability. Molecular Biology and Evolution 30: 772-780.

Maddison, D. \& Maddison, W. (2005) MacClade $v$. 4.08. Sunderland, Massachusetts: Sinauer Associates.

McCarthy, P. M. \& Elix, J. A. (2016) A new species of Micarea (lichenized Ascomycota, Pilocarpaceae) from alpine Australia. Telopea 19: 31-35.

Meyer, B. \& Printzen, C. (2000) Proposal for a standardized nomenclature and characterization of insoluble lichen pigments. Lichenologist 32: 571-583.

Miadlikowska, J., Kauff, F., Högnabba, F., Oliver, J. C., Molnár, K., Fraker, E., Gaya, E., Hafellner, J., Hofstetter, V., Gueidan, C. et al. (2014) A multigene phylogenetic synthesis for the class Lecanoromycetes (Ascomycota): 1307 fungi representing 1139 infrageneric taxa, 317 genera and 66 families. Molecular Phylogenetics and Evolution 79: 132-168.

Miller, M. A., Pfeiffer, W. \& Schwartz, T. (2010) Creating the CIPRES Science Gateway for inference of large phylogenetic trees. In Proceedings of the Gateway Computing Environments Workshop (GCE), 14 November 2010, New Orleans, Louisiana, pp.1-8.
Orange, A., James, P. W. \& White, F. J. (2010) Microchemical Methods for the Identification of Lichens (2nd edition). London: British Lichen Society.

Rambaut, A. (2009) FigTree v1.2.3. Available from: http://tree.bio.ed.uk/software/figtree/

Sérusiaux, E., Brand, A. M., Motiejunaite, J., Orange, A. \& Coppins, B. J. (2010) Lecidea doliiformis belongs to Micarea, Catillaria alba to Biatora, and Biatora ligni-mollis occurs in Western Europe. Bryologist 113: 333-344.

Stamatakis, A. (2006) RAxML-VI-HPC: maximum likelihood-based phylogenetic analyses with thousands of taxa and mixed models. Bioinformatics 22: 2688-2690.

Stamatakis, A., Hoover, P. \& Rougemont, J. (2008) A rapid bootstrap algorithm for the RAxML web servers. Systematic Biology 57: 758-771.

Svensson, M. \& Thor, G. (2011) Micarea capitata, a new bryophilous lichen from Sweden. Lichenologist 43: 401-405.

van den Boom, P. P. G. (2010) Lichens and lichenicolous fungi from Lanzarote (Canary Islands), with the descriptions of two new species. Cryptogamie, Mycologie 31: 183-199.

van den Boom, P. P. G. \& Ertz, D. (2014) A new species of Micarea (Pilocarpaceae) from Madeira growing on Usnea. Lichenologist 46: 295-301.

Zoller, S., Scheidegger, C. \& Sperisen, C. (1999) PCR primers for the amplification of mitochondrial small subunit ribosomal DNA of lichen-forming ascomycetes. Lichenologist 31: 511-516. 